\title{
Associação entre dieta enteral prescrita versus infundida e desfecho clínico em idosos internados em uma Unidade de Terapia Intensiva
}

\author{
Association between prescribed enteral diet and infused versus and clinical outcome in elderly stays \\ in an Intensive Care Unitasociación entre dieta enteral
}

Prescrita e infusión versus y resultado clínico en estadías de ancianos en Unidad de Cuidados Intensivos

Camila Melo de Araújo ORCID: https://orcid.org/0000-0003-2491-6294 Hospital Universitário Lauro Wanderley, Brazil E-mail: camilamelonutricao@ hotmail.com Adriana Gomes Cézar de Carvalho ORCID: https://orcid.org/0000-0002-6667-3550 Hospital Universitário Lauro Wanderley, Brazil E-mail: adricav04@gmail.com

Gina Araújo Martins Feitosa ORCID: https://orcid.org/0000-0002-9528-7037 Hospital Universitário Lauro Wanderley, Brazil E-mail: ginaamfeitosa@gmail.com

Caroline Sousa Cabral

ORCID: https://orcid.org/0000-0002-7899-7846 Hospital Universitário Lauro Wanderley, Brazil E-mail: carolinescabral@gmail.com

Janine Maciel Barbosa

ORCID: https://orcid.org/0000-0002-3622-8715 Hospital Universitário Lauro Wanderley, Brazil E-mail: janinebarbosa@gmail.com Edcleide Oliveira dos Santos Olinto ORCID: https://orcid.org/0000-0002-2680-4635 Hospital Universitário Lauro Wanderley, Brazil E-mail: edcleideoliveira@hotmail.com

Juliana Andrade da Silva ORCID: https://orcid.org/0000-0003-2836-9443 Hospital Universitário Lauro Wanderley, Brazil

E-mail: Juandradenutri06@hotmail.com

Paloma Egídio Andrade de Souza ORCID: https://orcid.org/0000-003-0258-978X Hospital Universitário Lauro Wanderley, Brazil E-mail: paloma.egidio@gmail.com

\begin{abstract}
Resumo
Introdução: O público idoso está em maior risco de desenvolver déficits nutricionais, uma vez que há um comprometimento da função fisiológica. Objetivo: avaliar a associação entre a dieta enteral prescrita versus infundida e o desfecho clínico de pacientes idosos internados em uma Unidade de Terapia Intensiva. Metodologia: O estudo foi seccional, desenvolvido com idosos em Unidade de Terapia Intensiva do Hospital Universitário Lauro Wanderley da Paraíba, onde foram avaliados através de uma ficha de acompanhamento nutricional. Na análise estatística utilizou-se os testes Qui-quadrado assumindo um nível de significância ( $\mathrm{p}<0,05)$. Resultados: Foram avaliados 79 idosos, sendo a maioria do sexo feminino com 62\%, e idade média de 72,5 anos. Quanto às intercorrências da terapia, a sonda nasogástrica aberta foi significativamente associada ao volume infundido versus prescrito não satisfatório. Pacientes cujo volume prescrito versus infundido foi satisfatório estiveram internados por mais de 21 dias. O total de 29 pacientes que tiveram alta hospitalar obteve $69 \%$, no qual, receberam satisfatoriamente o volume prescrito versus infundido. Por outro lado, 21 desses indivíduos apresentaram 63,6\% do total de óbitos que estiveram associados ao não atendimento desta variável. Conclusão: Observou-se que pacientes que permaneceram mais dias na UTI tiveram maior volume de dieta infundida alcançando suas metas preconizadas, em virtude daqueles que apresentou alta, notando-se que a maioria recebeu satisfatoriamente volume prescrito versus infundido, uma vez que é necessária a qualidade em terapia nutricional de forma mais ampla na população idosa.
\end{abstract}

Palavras-chave: Nutrição enteral; Idoso; Unidade de Terapia Intensiva. 


\begin{abstract}
Introduction: The elderly public is at greater risk of developing nutritional deficits, since there is an impairment of physiological function. Objective: to evaluate the association between the prescribed versus infused enteral diet and the clinical outcome of elderly patients admitted to an Intensive Care Unit. Methodology: The study was sectional, developed with elderly in Intensive Care Unit of the Hospital Universitário Lauro Wanderley da Paraíba, where they were evaluated through a nutritional monitoring form. Statistical analysis used Chi-square tests assuming a significance level $(\mathrm{p}<0.05)$. Results: Seventy-nine elderly patients were evaluated, being the majority female with $62 \%$, and mean age of 72.5 years. As for therapy complications, open nasogastric tube was significantly associated with unsatisfactory infused versus prescribed volume. Patients whose prescribed versus infused volume was satisfactory were hospitalized for more than 21 days. A total of 29 patients were discharged from the hospital, $69 \%$ of whom received satisfactory prescribed versus infused volume. On the other hand, 21 of these individuals had $63.6 \%$ of the total deaths that were associated with non-compliance with this variable. Conclusion: It was observed that patients who stayed more days in the ICU had a higher volume of infused diet reaching their recommended goals, in virtue of those who were discharged, noting that the majority satisfactorily received prescribed versus infused volume, since quality in nutritional therapy is needed more broadly in the elderly population.
\end{abstract}

Keywords: Enteral nutrition; Elderly; Intensive Care Unit.

\title{
Resumen
}

Introducción: El público en general tiene un mayor riesgo de desarrollar déficits nutricionales, ya que existe un deterioro de la función fisiológica. Objetivo: evaluar la asociación entre dieta enteral prescrita versus dieta enteral infundida y / o pérdida clínica de pacientes a largo plazo ingresados en la Unidad de Cuidados Intensivos. Metodología: Estudio seccional, desarrollado por personas de la Unidad de Cuidados Intensivos del Hospital Universitario Lauro Wanderley da Paraíba, donde se registran a través de un formulario de soporte nutricional. El análisis estadístico utilizó las pruebas de Chi-cuadrado, asumiendo un nivel de significancia ( $\mathrm{p}<0.05)$. Resultados: se evaluaron 79 personas, la mayoría mujeres, 62\%, y la edad promedio fue de 72,5 años. En cuanto a las complicaciones de la terapia, la sonda nasogástrica abierta se asoció significativamente con el volumen infundido frente a la prescripción insatisfactoria. Pacientes cuyo volumen prescrito versus volumen infundido fue satisfactorio para una estancia hospitalaria de más de 21 días. Un total de 29 pacientes que fueron dados de alta del hospital obtuvieron el $69 \%$, no los cuales recibieron satisfactoriamente o prescribieron versus volumen infundido. Por otro lado, 21 defunciones individuales correspondieron al 63,6\% del total de defunciones asociadas o no a esta variable. Conclusión: Observo que los pacientes que permanecen más tiempo en la UCI tendrán un mayor volumen de dieta infundida alcanzando sus objetivos recomendados, debido a darles que parecen altos, recordando que la mayoría de ellos recibieron un volumen prescrito satisfactorio versus infundido, ya que sería La calidad en la terapia nutricional es más necesaria para la población popular.

Palabras clave: Nutrición enteral; Anciano; Unidad de Terapia Intensiva.

\section{Introdução}

O estado nutricional dos indivíduos possui direta relação com uma inadequada ingestão, absorção e utilização de nutrientes no geral. As alterações decorrentes do envelhecimento associado ao uso contínuo de medicamentos acentuam esse quadro. O público idoso está em maior risco de desenvolver déficits nutricionais, uma vez que há um comprometimento da função fisiológica, redução de massa magra e da taxa metabólica basal, alterações sensoriais, complicações cardíacas e respiratórias maior número de internações, infecções e úlceras por pressão (Lew et al., 2017; Silva, Mannarino \& Moreira, 2014).

No contexto da terapia intensiva, essa realidade é ainda mais desafiadora, visto que a gravidade do quadro clínico dos pacientes, associado ao constante uso de antibióticos e opiodes corrobora com pior tolerância à dieta ofertada. Neste sentido, torna-se emergenciais medidas de enfrentamento da desnutrição hospitalar, especialmente no contexto da unidade terapia intensiva (Toledo \& Castro, 2015).

O prognóstico clínico do paciente crítico está intimamente relacionado com a realização de um suporte nutricional adequado (Cunha, Salluh \& França, 2010). Uma oferta de nutrientes adequada, especialmente de calorias e proteínas, é essencial na redução da incidência da desnutrição hospitalar, possibilitando a redução da morbidade e mortalidade desta clientela. (Graciano \& Ferretti, 2008). 
O monitoramento da terapia nutricional nas UTI tem sido amplamente discutido na prática clínica. No Brasil, essa avaliação contínua é possível por meio da utilização dos Indicadores de Qualidade de Terapia Nutricional (IQTN). Tais parâmetros contribuem para a gestão da qualidade da terapia nutricional, visando melhores desfechos clínicos do paciente (Dan, 2018).

Considerando a importância do aporte proteico-calórico adequado, um dos indicadores relacionados a essa gestão de qualidade é a avaliação da taxa de adequação do volume infundido em relação ao prescrito em pacientes em uso de terapia nutricional enteral. A avaliação desse parâmetro é fundamental para que o nutricionista possa avaliar se o seu planejamento em relação à oferta proteico-calórica foi alcançado ou não. Essa compreensão é fundamental, visto que a identificação dos fatores que dificultam a progressão da dieta garante ao profissional o melhor manejo clínico do paciente, de modo a traçar medidas corretivas necessárias à garantia do aporte calórico-proteico adequado (Dan, 2018).

Tendo em vista as alterações e imprevistos que acontecem em uma UTI durante o acompanhamento de um paciente, esta pesquisa tem como objetivo avaliar a associação entre a dieta enteral prescrita versus infundida e o desfecho clínico de pacientes idosos internados em uma Unidade de Terapia Intensiva.

\section{Metodologia}

Trata-se de um estudo de coorte transversal desenvolvido com a população idosa com idade igual ou superior a 60 anos, cujo tempo de permanência na unidade de terapia intensiva fosse igual ou superior a 72 horas e que faziam uso da terapia nutricional enteral internados em um Hospital Universitário localizado no município de João Pessoa. A amostra foi composta por 79 formulários de pacientes idosos internados no período de março de 2018 a março de 2019. A construção deste instrumento foi realizada a partir dos estudos e referências bibliográficas sobre terapia nutricional em pacientes críticos, bem como da experiência do pesquisador com a assistência nutricional em pacientes internados em unidades de terapia intensiva, utilizando como referência modelo proposto por (Sobotka et al. 2011). Para possibilitar a análise de associação, algumas variáveis foram categorizadas da seguinte forma: As intercorrências associadas à Terapia Nutricional foram categorizadas como presentes e ausentes. No que diz respeito ao desfecho clínico, foram criadas as categorias de alta hospitalar e óbito. Com relação ao tempo de internação foi categorizado adotando-se como ponto de corte o momento da internação em que esses pacientes tendem a se tornar crônicos $<21$ dias e $>21$ dias (Toledo \& Castro, 2015). O tempo de seguimento dos pacientes acompanhados nessa pesquisa foi equivalente ao tempo de internação na UTI.

\section{Analise estatística}

Os dados foram tabulados no Software Microsoft Excel® e analisados através do SPSS versão 13.0. Em seguida, foram utilizados os testes Qui-quadrado correção de continuidade e Teste exato de Fisher. Foram realizadas análises descritivas das variáveis do estudo por meio da identificação de frequências absolutas e relativas dos dados, bem como das medidas de tendência central e dispersão apropriadas, assumindo-se um nível de significância $\mathrm{p}<0,05$ como valor de referência para a significância estatística. A pesquisa atendeu todos os requisitos e foi aprovado pelo Comitê de Ética em Pesquisa do Hospital Universitário Lauro Wanderley da Universidade Federal da Paraíba, conforme parecer nº 3.449.341. Todos os indivíduos foram esclarecidos quanto aos procedimentos do estudo e assinaram o Termo de Consentimento Livre e Esclarecido.

\section{Resultados e Discussão}

Foram avaliados dados secundários dos prontuários de 79 pacientes idosos internados na unidade de terapia intensiva com terapia nutricional enteral exclusiva, por pelo menos 72 horas, de ambos os sexos, sendo a maioria do gênero feminino (62\%), com média de idade de 72,5 anos e desvio-padrão (9,4). O tempo de permanência variou entre 3 e 57 dias, com 
mediana de 14 dias (intervalo interqualítico de 8 -20 dias). O principal desfecho clínico encontrado foi a alta hospitalar (55,7\%). Quanto ao estado nutricional, em relação ao Índice de Massa Corporal e Circunferência do Braço, observou-se prevalência de baixo peso $(43,7)$ e desnutrição $(57.8 \%)$, respectivamente. Referente à nutrição enteral, 54,7\% dos pacientes atingiram volume prescrito. O tempo médio de internação foi de 17,35 dias $( \pm 12,42)$, enquanto que o tempo médio de uso da terapia nutricional enteral foi de 12,46 dias $( \pm 10,98)$. Os dados descritivos relacionados às características encontram-se disponíveis na Tabela 1.

Tabela 1. Características demográficas e nutricionais de pacientes em uso de TNE em uma UTI. João Pessoa, 2019.

\begin{tabular}{|c|c|c|}
\hline Variável & $\mathbf{N}$ & $\%$ \\
\hline \multicolumn{3}{|l|}{ Sexo } \\
\hline Masculino & 30 & $38,0 \%$ \\
\hline Feminino & 49 & $62,0 \%$ \\
\hline \multicolumn{3}{|l|}{ Idade } \\
\hline $60-69,9$ & 34 & $43,0 \%$ \\
\hline 70-79,99 & 28 & $35,4 \%$ \\
\hline$>=80$ & 17 & $21,5 \%$ \\
\hline \multicolumn{3}{|l|}{ Estado nutricional (IMC) } \\
\hline Baixo peso & 31 & $43,7 \%$ \\
\hline Eutrofia & 25 & $35,2 \%$ \\
\hline Sobrepeso & 6 & $8,5 \%$ \\
\hline Obesidade & 9 & $12,7 \%$ \\
\hline \multicolumn{3}{|l|}{ Estado nutricional ( CB) } \\
\hline Desnutrido grave/moderado & 13 & $28,9 \%$ \\
\hline Desnutrido leve & 13 & $28,9 \%$ \\
\hline Eutrofia & 14 & $17,7 \%$ \\
\hline Sobrepeso & 2 & $2,5 \%$ \\
\hline Obesidade & 3 & $3,8 \%$ \\
\hline Tempo de internamento & \multicolumn{2}{|c|}{17,35 dias $\pm 12,42$} \\
\hline Tempo de uso da TN & \multicolumn{2}{|c|}{12,46 dias $\pm 10,98$} \\
\hline
\end{tabular}

$\mathrm{CB}=$ circunferência do braço; $\mathrm{IMC}=$ índice de massa corporal; $\mathrm{TN}=$ terapia nutricional. Fonte: Autores.

Com relação às intercorrências relacionadas à Terapia Nutricional, a constipação foi mais frequente entre os indivíduos avaliados $(62 \%)$, seguida de diarreia, $(34,6 \%)$, sonda aberta $(32,9 \%)$ e vômito $(16,5 \%)$. A tabela 02 demonstra a associação dessas intercorrências em relação ao indicador Volume Prescrito versus infundido. 
Tabela 2. Associação do indicador volume prescrito versus infundido e as intercorrências relacionadas à Terapia Nutricional. João Pessoa, 2019.

\begin{tabular}{|c|c|c|c|c|}
\hline \multirow{2}{*}{\multicolumn{2}{|c|}{ Intercorrência }} & \multicolumn{2}{|c|}{ Volume prescrito versus infundido } & \multirow{2}{*}{ p-valor } \\
\hline & & Satisfatório & Não satisfatório & \\
\hline \multirow[t]{3}{*}{ Constipação } & & & & 0,281 \\
\hline & Presente & $29(59,2 \%)$ & $20(40,8 \%)$ & \\
\hline & Ausente & $12(46,2 \%)$ & $14(53,8 \%)$ & \\
\hline \multirow[t]{3}{*}{ Diarreia } & & & & 0,613 \\
\hline & Presente & $16(59,3 \%)$ & $11(40,7 \%)$ & \\
\hline & Ausente & $25(53,2 \%)$ & $22(46,8 \%)$ & \\
\hline \multirow[t]{3}{*}{ SNG Aberta } & & & & 0,007 \\
\hline & Presente & $8(32,0 \%)$ & $17(68,0 \%)$ & \\
\hline & Ausente & $30(65,2 \%)$ & $16(34,8 \%)$ & \\
\hline \multirow[t]{3}{*}{ Vômito } & & & & 0,498 \\
\hline & Presente & $6(46,2 \%)$ & $7(53,8 \%)$ & \\
\hline & Ausente & $35(56,5 \%)$ & $27(46,5 \%)$ & \\
\hline
\end{tabular}

Const= Constipação Dr=Diarreia SNG= Sonda nasogastrica VO= Vomito. Fonte: Autores.

De acordo com os dados expressos na Tabela 2, o uso de sonda nasogástrica aberta esteve significativamente associado ao volume prescrito versus infundido não satisfatório. Do total de pacientes que apresentaram essa intercorrência, $68 \%$ não tiveram o volume infundido de acordo com o que foi prescrito $(\mathrm{p}=0,007)$. Com relação às demais intercorrências, nenhuma delas esteve significativamente associada ao não atendimento desse indicador.

A Tabela 3 apresenta uma análise da associação entre o atendimento ou não do indicador volume prescrito versus infundido em relação aos respectivos desfechos clínicos, bem como em relação aos dias de internação.

Tabela 3. Associação entre indicador volume prescrito versus infundido com dias de internação e desfecho clínico em hospital universitário de João Pessoa - PB, 2019.

\begin{tabular}{llll}
\hline & \multicolumn{2}{l}{ Volume prescrito versus infundido } \\
& Satisfatório & Não satisfatório & p-valor \\
\hline $\begin{array}{l}\text { Tempo de Internação em UTI } \\
\text { (dias) }\end{array}$ & Md(IQ) & Md(IQ) & 0,014 \\
$\leq 21$ dias & $26(46,4 \%)$ & $30(53,6 \%)$ & \\
$>21$ dias & $15(78,9 \%)$ & $4(21,1 \%)$ & 0,010 \\
Desfecho & & $13(31,0 \%)$ & \\
Alta & $29(69,0 \%)$ & $21(63,6 \%)$ & \\
Óbito & $12(36,4 \%)$ & \\
\hline
\end{tabular}

Md: Mediana; IQ: intervalo interquartílico; UTI: unidade de terapia intensiva U; ${ }^{* *}$ Qui quadrado - correção de continuidade; ${ }^{* * *}$ Teste exato de Fisher. Fonte: Autores.

Os dados demonstraram associação significativa entre as variáveis avaliadas. Com relação ao tempo de internação, $15(78,9 \%)$ pacientes cujo volume prescrito versus infundido foi satisfatório estiveram internados por mais de 21 dias $(\mathrm{p}=0,014)$. Avaliando-se o desfecho, do total de 29 pacientes que tiveram alta hospitalar com 69,0\% receberam 
satisfatoriamente o volume prescrito versus infundido. Por outro lado, 21 desses indivíduos teve $63,6 \%$ do total de óbitos que estiveram associados ao não atendimento desse indicador $(\mathrm{p}=0,0103$

Em relação aos indicadores de estado nutricional a CB apresentou maior frequência de desnutrição e o IMC de baixo peso. Martins et al. (2017) encontraram, no tocante a CB, que a maioria dos pacientes estava desnutrida, estando consonante com o presente estudo. A falta de detecção precoce do estado nutricional se configura como um fator de risco para mortalidade, cicatrização mais lenta de feridas, aumento no tempo de internação hospitalar e aumento no número de complicações e com isso, maiores custos relacionados ao tratamento de doentes hospitalizados. (Stefanello \& Poll, 2014).

Quanto ao indicador volume prescrito $\mathrm{x}$ infundido, não se observou o alcance da meta proposta pela ILSI (International Life Sciences Institute), que deve ser $\geq$ a 70\%. Dados semelhantes também foram encontrados por Ypi, Rai e Wong (2014), em um estudo realizado com 77 pacientes em ventilação mecânica em nutrição enteral, no qual, 66\% dos pacientes atingiram $80 \%$ das necessidades calóricas, podendo ter como causas interrupções da dieta, fazendo com que haja uma oferta aquém das reais necessidades, podendo ser deletéria e incorrendo com redução de massa magra.

O estudo de Ribeiro et al. (2014) mostrou que as pausas para extubação somadas àquelas por complicações gastrointestinais, problemas relacionados à sonda e procedimentos de rotina somam $78,1 \%$ do total de horas em pausa, podendo destacar que as causas internas à unidade foram as responsáveis pelas interrupções da terapia nutricional enteral, tornando fundamental a mobilização de toda a equipe, a fim, de evitar as causas. Os mesmos autores também destacaram as características próprias do paciente, como a idade, sendo determinante na ocorrência de morbidades e mortalidade, mostrando que idosos com mais de 60 anos apresentam porcentagem de recuperação da via oral inferior a indivíduos mais jovens, isso pode ser explicado pelo fato de que esses pacientes têm maior risco de disfagia pós-extubação, o que estende o uso da via oral para alimentação, tendo como consequência o prolongamento no uso da dieta enteral e possível impacto no volume de dieta efetivamente infundida.

Quanto à análise das intercorrências gastrointestinais constipação, diarreia, sonda nasogástrica aberta e vômito, achados semelhantes foram encontrados no estudo de Medeiros et al. (2020) onde as complicações relacionadas à nutrição enteral foram evidenciadas em $65,3 \%$ dos pacientes, sendo a constipação a mais predominante $(20,8 \%)$ seguida de diarreia $(18,1 \%)$. A literatura mostra que alguns fatores influenciam na adequação da oferta de volume infundido, como as causas internas (complicações gastrointestinais, extubação, problemas referentes à sonda, procedimentos de rotina e reintrodução de nutrição enteral) e externas (jejum para tomografia, broncoscopia, endoscopia digestiva alta ou traqueostomia (Telles, Mariano \& Paula, 2015).

Não foram observadas associações entre a maioria das intercorrências à TNE e o volume prescrito versus infundido, exceto para SNG aberta, que foi significativamente associada ao volume prescrito versus infundido não satisfatório, mostrando que outros fatores externos que não propriamente intercorrências gastrintestinais podem estar relacionados ao não alcance ideal do indicador volume prescrito versus infundido, se assemelhando ao estudo de Choi, Park e Park ( 2014) no qual dos 308 motivos relacionados a não administração da dieta, tiveram maior representatividade os referentes a cálculo errado da velocidade de infusão praticado pela equipe de enfermagem, realização de exames diagnósticos ou terapêuticos, procedimentos cirúrgicos e tentativa de intubação e extubação.

Dos motivos relacionados à não administração da dieta, alguns apresentam maior representatividade como os referentes à cálculo errado da velocidade de infusão praticado pela equipe de enfermagem, realização de exames diagnósticos e terapêuticos, procedimentos cirúrgicos e tentativa de intubação e extubação. Fatores como ausência de justificativa para o evento, obstrução ou deslocamento da sonda, introdução da bomba de insulina e falha ou atraso na entrega da NE pelo serviço de nutrição também são motivos encontrados, porém, com menor frequência. Sendo assim, os motivos evitáveis, ou seja, os 
passíveis de prevenção, apresentaram maior representatividade em relação aos não evitáveis, incorrendo na não administração do volume de dieta enteral prescrita (Filho, et al., 2016).

Santos e Alves (2018) em estudo longitudinal, quantitativo, realizado em um hospital público de Salvador (BA), no qual, foram incluídos 51 pacientes adultos e idosos recebendo terapia nutricional enteral exclusiva, encontraram que os pacientes com menor quantidade de intercorrências cursaram com volumes médios de ingestão entre 90 e 100\%. Nesse mesmo estudo, um 70,2\% dos pacientes receberam volume inferior a $80 \%$ do volume prescrito pelo nutricionista, o que refletiu em piora do estado nutricional. Outros achados dos autores mostram que as principais causas para não recebimento da dieta conforme prescrito são problemas logísticos, estase gástrica e perda acidental da sonda.

Em um estudo com 155 pacientes com média de idade de 73 anos (Vieira, 2019) analisou valores de infusão diária da nutrição enteral em 5 a 10 dias, buscando investigar a associação da adequação calórica através de um ponto de corte ( > 70\% da prescrição e $<70 \%$ ) com os desfechos. Ao comparar os pacientes que receberam mais de $70 \%$ da dieta com os que receberam menos de 70\%, os primeiros apresentaram menor número de óbitos em 30 dias. Feitosa et al. (2020) semelhantemente ao presente estudo, obtiveram uma associação significativa, mostrando que os pacientes que receberam adequadamente o volume prescrito cursaram com alta hospitalar.

O tempo de internação pode afetar a oferta de energia, uma vez que pacientes com estadias mais longas tendem a atingir uma maior adequação relativa à meta proposta de calorias. Isso porque nos primeiros dias os pacientes tendem a apresentar quadros mais instáveis, ou seja, alguns estudos mostram que a alimentação hipocalórica traz efeitos benéficos sobre os resultados clínicos, pois representaria menor carga fisiológica e menor risco de supressão da autofagia para os pacientes de UTI, considerando ainda que a alimentação se torna mais difícil em pacientes graves, visto que não podem tolerar maior ingestão calórica (Couto, et al., 2019).

Choi, Park e Park (2014) em uma metanálise de ensaios clínicos randomizados apontam que até o momento não há uma ingestão ideal de calorias de NE para pacientes críticos, ressaltando que a literatura fornece dados conflitantes e recomendações tanto para suporte completo de calorias como para subalimentação permissiva. Neste contexto, os estudos mostram que a subalimentação de pacientes gravemente enfermos pode levar a desnutrição e terá como consequência vários efeitos adversos, particularmente as infecções, maior duração na ventilação mecânica e perda muscular. Sendo assim, o aumento da ingestão pode estar associado à redução da mortalidade em pacientes graves.

Uozumi et al., (2017), em um estudo com 85 pacientes adultos em UTI durante 30 dias do início do uso de nutrição enteral até a sua suspensão, apontam que no grupo que apresentou óbito como desfecho clínico houve uma inadequação do volume infundido e prescrito (< 80\%), estando em consonância com o presente estudo. No estudo de Patkova et al. (2017), realizado com pacientes adultos que receberam terapia nutricional enteral por mais de 72 horas, em que foram estabelecidas como metas os valores de $<70,70-90 \%$, $>90 \%$, foi encontrado um maior número de óbito naqueles com um consumo inferior a70\% Como limitações da pesquisa, temos o reduzido número da amostra e estudos científicos mais expressivo para comprovarmos a associação entre dieta enteral prescrita versus infundida e desfecho clinico em idosos internados em uma unidade de terapia intensiva.

\section{Conclusão}

Este estudo concluiu que a intercorrência gastrointestinal sonda aberta se associou a uma inadequada oferta de dieta enteral, observando ainda que pacientes que permaneceram mais dias na UTI tiveram maior volume de dieta infundida alcançando suas metas preconizadas. A literatura ainda mostra controvérsias sobre a oferta ideal de infusão de dietas em indivíduos gravemente enfermos, por outro lado, é importante considerar que a hipoalimentação pode ser viável nos primeiros dias, visto quadro de gravidade, na medida em que esses pacientes se tornam estáveis e crônicos, uma maior oferta se constitui 
como ideal para prevenção de desnutrição. Em virtude daqueles que apresentaram alta, notou-se que a maioria recebeu satisfatoriamente volume prescrito versus infundido, mostrando que a mortalidade pode estar associada ao não atendimento deste indicador, uma vez que é necessária a qualidade em terapia nutricional de forma mais ampla para melhorar a avaliação da assistência nutricional de pessoas idosas em UTI.

\section{Referências}

Choi, E. Y., Park, D., \& Park, J. (2014). Calorie intake of enteral nutrition and clinical outcomes in acutely critically ill patients: A meta-analysis of randomized controlled trials. Journal Parenteral and Enteral Nutrition, 20(10).

Cunha, H. F. R.; Salluh, J. I. F., \& França, M. F. (2010). Atitudes e percepções em terapia nutricional entre médicos intensivistas: um inquérito via internet. Rev Bras Ter Intensiva. 22 (1), 53-63.

Couto, C. F. L.; Dariano, A.; Teixeita, C.; Silva, C. H.; Torbes, A. B.; \& Friedman, G. (2019). A adequação de suporte nutricional enteral em unidades de terapia intensiva não afeta o prognóstico a curto e longo prazo de pacientes sob ventilação mecânica: um estudo piloto. Rev Bras de Ter Intensiva. 31(1):34-38.

Filho, R. S. O., Ribeiro, L. M. K., Caruso, L., Lima, P. A., Damasceno, N. R. T., \& Soriano, F. G. (2016). Indicadores de qualidade para terapia nutricional enteral e parenteral: aplicações em pacientes críticos em risco nutricional'. Nutricion Hospitalaria. 33(5):1027-1035.

Graciano, R. D. M., \&Ferreti, R. E. L. (2008). Nutrição enteral em idosos na unidade de terapia intensiva: prevalência e fatores associados. Geriatria $e$ Gerontologia, 2(4), 151-155.

Indicadores de qualidade em terapia nutricional: 10 anos de IQTN no Brasil resultados, desafios e propostas. (2018) / [coordenação científica Dan LinetzkyWaitzberg]. (3a ed.).

Lew, C. C. H.; Yandell, R., Fraser, R. J., Chua, A. P., Chong, M. F. F., \& Miller, M. (2017). Associação entre desnutrição e resultados clínicos na unidade de terapia intensiva: uma revisão sistemática. JPEN J Parenter Nutr Enteral. 41 (5): 744-758.

Martins, F. C.; Cristina, R.; Vital, C.; Wendel Amaral, F.; Volp, J. P.; \& Carolina, A. (2017). Perfil nutricional de pacientes internados em terapia intensiva. Nutr. clin. diet. Hosp.37(4):40-47.

Medeiros, I. M. S; Ritter, C. G; Filho, G. H. C. R.; \& Prado, P. R. (2020). Caracterização e adequação energético-proteica da nutrição enteral em pacientes em uma unidade de terapia intensiva. South American Journal, 6(2):381-395.

Patckova, A; Joskova, V; Havel, E; Kovarik, M; Kuckarova, M; Zadak, Z; \& Hronek, M. (2017). Energy, Protein, Carbohydrate, and Lipid Intakes and Their Effects on Morbidity and Mortality in Critically Ill Adult Patients: A Systematic Review. American Society for Nutrition . 8: 624-34.

Ribeiro, L. M. K.; Filho, S. R. O.; Caruso, L.; Lima, P. A.; Damasceno, N. R. T.; \& Soriano, F. G. (2014). Adequação dos balanços energético e proteico na nutrição por via enteral em terapia intensiva: quais os fatores limitantes? Rev Bras Ter Intensiva, 26(2), 155 - 162.

Stefanello, M. D., \& Poll, F. A. Estado nutricional e dieta enteral prescrita e recebida por pacientes de uma Unidade de Terapia Intensiva. ABCS Health Sciences. 39(2):71- 76 .

Santos, A. L; \& Alves, T. C. H. S. (2018). Terapia nutricional enteral: relação entre percentual de dieta prescrito e administrado e intercorrências associadas em hospital público de Salvador-BA. Braspen J, 33(1): p 58-63.

Silva, A. S; Mannarino I. S., \& Moreira, A. S. B. (2014). Risco nutricional em pacientes idosos hospitalizados como determinantes de desfecho clínicos. Revista Geriatria e Gerontologia. 8 (1), 32-37. (4a ed.).

Telles, J. L. H.; Boton, C. R. M.; Mariano, M. L. L., \& Paula, M. A. B. (2015). Nutrição enteral: complicações gastrintestinais em pacientes de uma unidade de terapia intensiva. Revista Científica de Enfermagem, 5(3), 5-11.

Toledo, D.; \& Castro, M. (2015). Terapia nutricional em terapia UTI,

Vieira, R. M. (2019). Valor nutricional administrado e impacto da oferta proteica calórica em pacientes cardíacos críticos em nutrição enteral. Dissertação Mestrado em ciências da saúde: cardiologia e ciências cardiovasculares.

Uozumi, M., Sanui, M., Komuro, T., Iizuka, Y., Kamio, T., Koyama, H., et al. Interruption of enteral nutrition in the intensive care unit: a single-center survey. J Intensive Care. 5:52.

Yip, K. F.; Rai, V., \& Wong, K. K. (2014). Evaluation of delivery of enteral nutrition in mechanically ventiladed Malaysian ICU patients. BMC Anesteshiology. 